\title{
ARDUINO BASED AUTOMATIC PLANT WATERING/IRRIGATION SYSTEM
}

\author{
Neha Singh \\ Department of ECE \\ Buddha Institute of Technology, Gorakhpur, \\ UP, India \\ Sana Mukhtar \\ Department of ECE \\ Buddha Institute of Technology, Gorakhpur \\ UP, India
}

\begin{abstract}
Irrigation In the present days, the farmers are suffering from severe drought like condition throughout the year. The main objective of this paper is to provide a system leads to automatic irrigation thereby saving time, money \& power of the farmers, gardeners in greenhouses etc. An adequate water supply is important for plant growth. When rainfall is not sufficient, the plants need additional water. We know that people do not pour the water on to the plants in their gardens when they go to vacation or often forget to water plants. As a result, there is a chance to get the plants damaged. This paper presents a technique for Arduino based Automatic Irrigation System. With this automated technology of irrigation, human intervention can be minimized.

The moisture sensors will be bed in on the field. Whenever there is a change in water concentration, these sensors will sense the change and gives an interrupt signal to the micro-controller. Soil is one of the most fragile resources whose soil pH property used to describe the degree of the acidity or basicity, which affects nutrient availability and ultimately plant growth. Thus, the system will provide automation, remote controlling and increased efficiency. Humidity sensor is connected to internal ports of microcontroller via comparator; [1] Sandeep Minz et al whenever there is a fluctuation in temperature and humidity of the environment, these sensors sense the change in temperature and humidity and give an interrupt signal to the micro-controller and thus the motor is activated.
\end{abstract}

Keywords - Arduino; Moisture Sensor; Automatic Irrigation; Xbee Introduction

\section{INTRODUCTION}

The main aim of this project was to provide water to the plants or gardening automatically using microcontroller (Arduino Uno). We can automatically watering the plants when we are going on vacation or don't we have to bother my

\author{
Shreya Srivastava \\ Department of ECE \\ Buddha Institute of Technology, Gorakhpur, \\ UP, India \\ Rahul Mani Upadhyay \\ Department of ECE \\ Buddha Institute of Technology, Gorakhpur \\ UP, India
}

neighbors, Sometimes the neighbors do too much of watering and the plants end up dying anyway. There are timer based devices available in India which waters the soil on set interval. They do not sense the soil moisture and the ambient temperature to know if the soil actually needs watering or not. Assimilation is that the artificial application of water to the land or soil It is used to assist in the growing of agricultural crops [3], maintenance of landscapes, and re vegetation of disturbed soils in dry areas and during periods of inadequate rainfall. K. Srikar et al When a zone comes on, the water flows through the lateral lines and ultimately finally ends up at the irrigation electrode (drip) or mechanical device heads. Several sprinklers have pipe thread inlets on the lowest of them that permits a fitting and also the pipe to be connected to them S. Borromeo et al (2011). The sprinklers are usually used in the top of the head flush with the ground surface [9]. As the method of dripping will reduce huge water losses it became a popular method by reducing the labor cost and increasing the yields. When the components are activated, all the components will read and gives the output signal to the controller, and the information will be displayed to the user (farmer). The sensor readings are analog in nature so the ADC pin in the controller will convert the analog signals into digital format [8] M.P. Durisic et al (2012). Then the controller will access information and when the motors are turned On/Off it will be displayed on the LCD Panel, and serial monitor windows [10] G Lopez et al (2010). There are many systems are available to water savings in various crops, from basic ones to more technologically advanced ones. For instance, in one system plant watering status was monitored and irrigation scheduled based on temperature presents in soil content of the plant.

\section{WORKING}

An automatic plant watering system using Arduino microcontroller UNO R3 is programmed such that it gives the interrupt signals to the motor via the motor driver module. Soil 


\section{International Journal of Engineering Applied Sciences and Technology, 2020 \\ Vol. 4, Issue 12, ISSN No. 2455-2143, Pages 273-276 \\ Published Online April 2020 in IJEAST (http://www.ijeast.com)}

sensor is connected to the A0 pin to the Arduino board which senses the moisture content present in the soil. Whenever the soil moisture content values goes down, the sensor senses the humidity change, giving signal to the microcontroller so that the pump (motor) can be activated. This concept can be used for automatic plant watering system. D. k. Fisher et al (2010) The circuit comprises an Arduino UNO board [2], a soil moisture sensor, a 5V motor pump, a Motor driver L293D (IC1), motor driver IC to run the water pump. You can power the Arduino board using a $5 \mathrm{~V}$ to $9 \mathrm{~V}$ wall wart or plug in adaptor or solar panel. You need a separate $5 \mathrm{~V}$ to 9vbattery for the pump motor.

\section{COMPONENT AND REQUIRNMENT}

\subsection{Arduino}

In figure. 1 it is showing an Arduino board is an open source platform used for building electronics projects. Arduino is a programmable circuit's board which we can write a program based on your projects. Arduino program will be uploading with IDE (Integrated Development Environment) software that runs on your computer, it is used to write and upload computer code to the Arduino physical board. Arduino language is merely a set of $\mathrm{C} / \mathrm{C}++$ functions that can be called from your code.

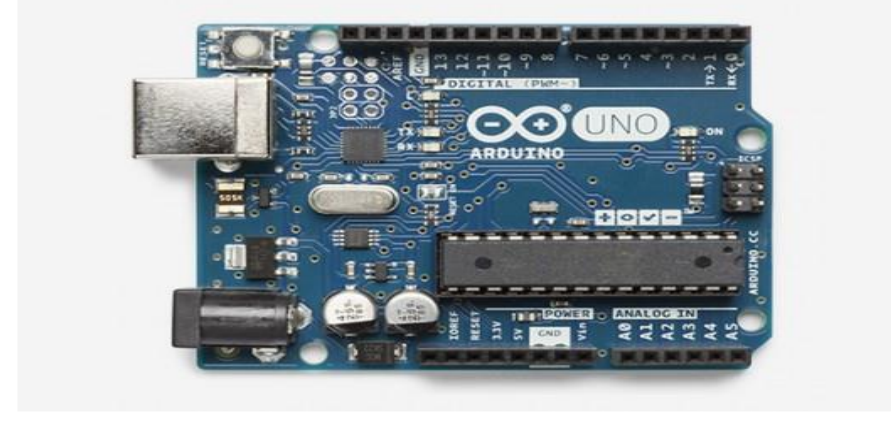

Fig.1 ARDUINO UNO BOARD

\subsection{Relay}

In figure 2, shows are a relay is an electrically operated switch. Several relays use a magnet to automatically operate a switch, however alternative in operation principles are used, like solid state relays. Relays are used wherever it's necessary to regulate a circuit by a separate low-power signal, or wherever many circuits should be controlled by one signal. [4]. R.Jaichandran et al (2013) The essential relays were handling in long distance communicate circuits as amplifiers, they unbroken the signal coming back in from one circuit and re-transmitted it on another circuit.

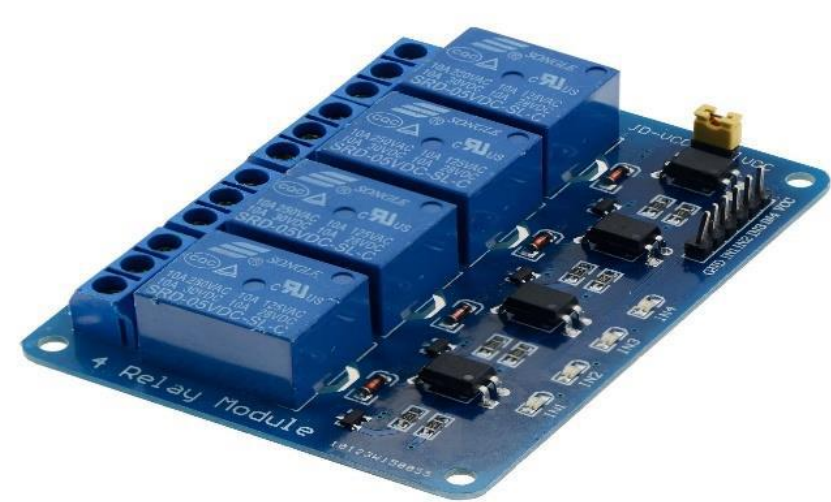

Fig.2 Relay

\subsection{Soil Sensor}

In figure 3, Soil moisture sensors measure the humidity of water content in soil. Since the direct hydrometric measuring of free soil wetness needs removing, during, and coefficient of a sample, soil wetness sensors live the meter water content indirectly by victimization another property of the soil, like electrical phenomenon, one conductor constant, or interaction with neutrons, as a proxy for the wetness content. [2]

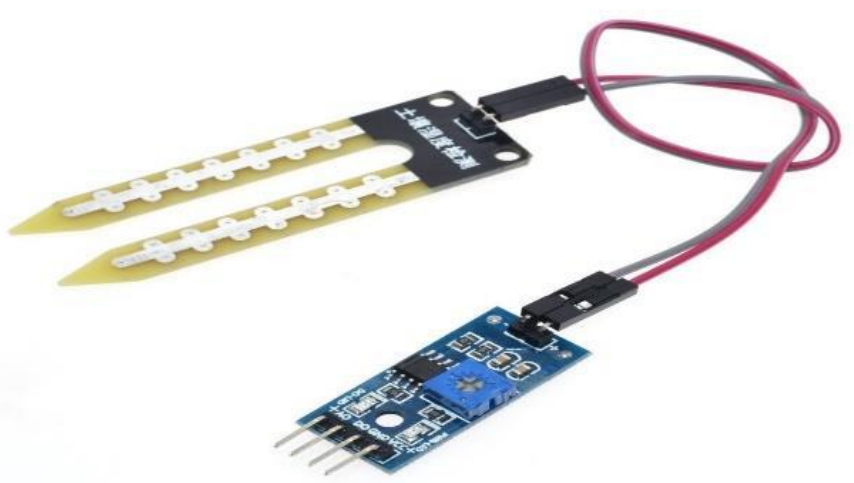

Fig.3 Soil Sensors

\subsection{Motor 5V}

An AC motor is an electrical motor driven by Associate in alternating current (AC). In figure: 5, The AC motor normally consists of two basic components, an outdoor stationary stator coil having coils furnished with AC to supply a rotating flux, and an indoor rotor connected to the output shaft manufacturing a second rotating flux. The rotor flux could also be made by permanent magnets, reluctance striking, or DC or AC electrical windings. 


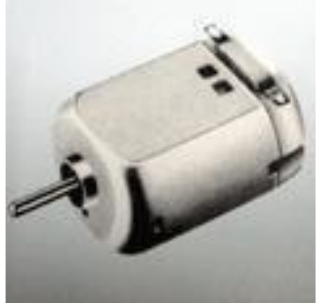

Fig.4 Motor

\subsection{Transistor}

The 2N2222 may be a common NPN bipolar semiconductor device; bipolar junction transistors (BJT) used for general purpose low-power amplifying or switch applications. It is designed for low to medium current, low amplifying current, low power, medium voltage, and might operate at moderately high speeds. It had been originally created within the TO-18 metal.

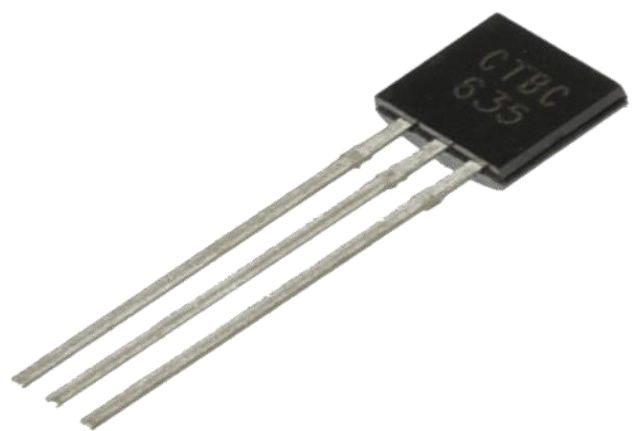

Fig.5 Transistor

\subsection{Resistor}

Here in figure 6 , it is an electrical device may be a passive two-terminal electrical part that implements resistance as a circuit component. Venkata Naga Rohit Gunturi (2013). In electronic circuits, resistors unit of measurement accustomed reduce current flow, alter signal levels, to divide voltages, bias active components, and terminate transmission lines, among completely different uses.[5]

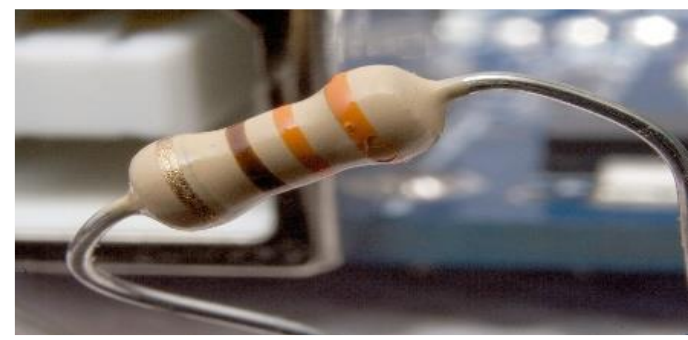

Fig.6 Resister

\subsection{Pipe}

Here it is used as a water channel, and pipe has been used for watering plant.

\section{BLOCK DIAGRAM}

The block diagram of the proposed system can be represented as shown in Figure.

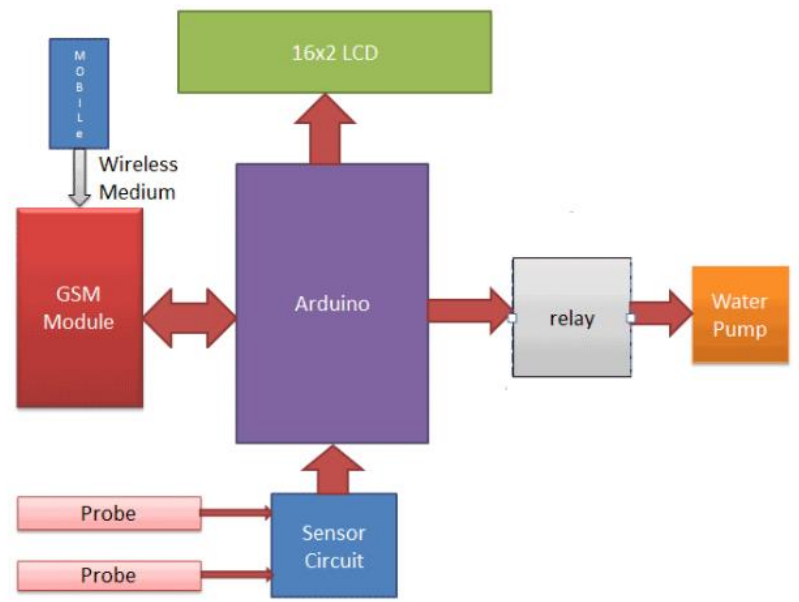

\section{ADVANTAGES}

- Saves water

- Improves growth

- Discourages weeds

- Saves time

- Helps control fungal diseases

- Adaptable

- Eliminates the manual operation of opening or closing valves

- Adaption of the advanced irrigation systems and the new technologies, especially the new irrigation systems that are complex and difficult to operate manually.

- The system will be operated in night also which results in minimization of the water loss due to evaporation.

- Irrigation process starts and stops exactly when required, thus optimizing energy requirements

\section{RESULTS}

Irrigation becomes easy, accurate and practical with the same soil sample impossible. Because of the idea above shared and can be implemented in agricultural difficulties of accurately measuring dry soil and water fields in future to promote 


\section{International Journal of Engineering Applied Sciences and Technology, 2020 Vol. 4, Issue 12, ISSN No. 2455-2143, Pages 273-276 \\ Published Online April 2020 in IJEAST (http://www.ijeast.com)}

agriculture to next level. [7]. S.D. Gainwar et al (2015). The Volumes, volumetric water contents are not usually output from moisture sensor and level stem plays major determined directly. Role in producing the output.

\section{CONCLUSION AND FUTURE SCOPE}

The Cultivation Management System based on cloud is a very effective system for the user, which cultivates the plants on the farm field by this farmer can examine their farm field information and detail from anywhere in between range. The proposed monitor tool gives soil moisture details, water level details which can help to increase in productivity by using such automated irrigation system.

The proposed system can be extended in future by adding the feature for remotely monitoring sensors that can detect crop growth and livestock feed levels. In the future, adding the features that can remotelymanage and control their smart connected other irrigation equipment in the proposed system can be investigated.

\section{ACKNOWLEDGMENT}

The Author would like to thank Buddha Institute of Technology Gorakhpur for providing all the facility of my research work.

\section{REFERENCES}

[1] Sandeep Minz "Arduino Based Automatic Plant Irrigation system” Proc AJEEE May 2019

Volume 3, Issue 1.

[2] D. K. Fisher and H. A. Kebede, -A low-cost microcontroller-based system to monitor crop

temperature and water status, $\|$ Comput. Electron. Agriculture., vol. 74, no. 1, pp. 168-173, Oct. 2010.

[3] K. Srikar, M.Akhil ,V.Krishna reddy," Execution of Cloud Scheduling Algorithms",International Innovative Research Journal of Engineering and Technology, vol 02, no 04,pp.108-

[4] R. Jaichandran, Dr. A. Anthony Irudhayaraj, Surabhi, Rajkumar Kuila, Trisha Sinha, "Prototype for Automatic Controlling and Remote Accessing of Irrigation Motor", International Journal of Innovative Research in Computer and Communication Engineering, 2013.

[5] Venkata Naga Rohit Gunturi, "Micro Controller Based Automatic Plant Irrigation System", International Journal of Advancement in Research and Technology, 2013
[6] A. N. Arvindan and D. Keerthika, "Experimental investigation of remote control via Android smart phone of arduino-based automated irrigation system using moisture sensor", Proc. of 2016 3rd International Conference on Electrical Energy Systems (ICEES), Chennai, 17-19 March 2016, pp. 168-175. Doi: 10.1109/ICEES.2016.7510636 $111,2017$.

[7] S. D. Gainwar and D. V. Rojatkar, "Soil Parameters Monitoring with Automatic Irrigation System”, International Journal of Science, Engineering and Technology Research (IJSETR), Vol. 04, Issue 11, Nov. 2015.

[8] M. P. Durisic, Z. Tafa, G. Dimic, and V. Milutinovic, -A survey ofmilitary applications of

wireless sensor networks, $\|$ inProc. MECO,Jun. 2012, pp. 196199.

[9] M. C. Rodríguez-Sánchez, S. Borromeo, and J. A. Hernández-Tamames, -Wireless sensor networks for conservation and monitoring cultural assets, \|IEEE Sensors J., vol. 11, no. 6, pp. 1382-1389, Jun. 2011.

[10] G. López, V. Custodio, and J. I. Morenoz -LOBIN: Etextile and wireless sensor network based

platform for healthcare monitoring in future hospital environments, \| IEEE Trans. Inf. Technol. Biomed., vol.14, no. 6, pp. 1446-1458, Nov. 2010.

[11] J. M. Corchado, J. Bajo, D. I. Tapia, and A. Abraham, monitoring system for healthcare,\| IEEE Trans. Inf. Technol. Biomed., vol. 14, no. 2,pp. 234- 240,Mar,2013

[12] Fang Meier, D.D., Garrote, D.J., Mansion, F. and S.H. Human. 1990. Automated Irrigation Systems Using Plant and Soil Sensors. In: Visions of the Future. ASAE Publication 0490. American Society of Agricultural Engineers, St. Joseph, Michigan, pp. 533-537.

[13] "Arduino Based Automatic Plant Watering System", S. V. Devika, S.k.Khamuruddeen, Sk.Khamurunnisa, Jayanth Thota, Khalesha Shaikh, Associate Professor, Dept. of ECE, HITAM,Hyderabad, India, MSC 2nd Year,

Department Of Electronics, HRD, Hyderabad, India. Websitewww.ijarcsse.com 